\title{
Constructing routing tree for centralized scheduling using multi-channel single transceiver system in 802.16 mesh mode
}

\begin{abstract}
The WiMAX mesh networks based on IEEE 802.16 standard was developed with the base station (BS) as a coordinator for centralized scheduling. However, interferences from transmission of the neighbouring nodes within the mesh networks are inevitable. This paper proposes a centralized scheduling algorithm that can reduce interferences by constructing routing tree with multi-channel single transceiver system in WiMAX mesh networks. In this algorithm, each node has one transceiver that can be tuned to any of the channels, intending to eliminate the secondary interference. The parameters of interference, hop-count, no. of children per node, spatial reuse, fairness, load balancing, quality of services (QoS) and node identifier (ID) are considered. The results of analysis show that this proposed algorithm greatly improves the length of scheduling and the channel utilization ratio (CUR).
\end{abstract}

Keyword: 802.16d; Routing tree; Centralized scheduling; Multi-channel single transceiver 